\title{
How Connectedness Contributes to Experimental Smoking Among Rural Youth: Developmental and Ecological Analyses
}

\author{
Michael J. Karcher ${ }^{1,2}$ and Laurel Finn ${ }^{1}$
}

The influences of peer, sibling, and parents' smoking on adolescents' initiation of tobacco use have been explained as a function of peer pressure, genetics, and social learning processes, but rarely in combination or with assessments of the quality of these relationships. This study examined the additional contributions of connectedness to friends, siblings, parents, and teachers beyond the effects of friend, sibling, and parental smoking using logistic regression analyses with a cross-sectional middle and high school sample of 303 rural adolescents. Friends' and siblings' smoking, and connectedness to friends, were the strongest predictors of experimental smoking. Parental smoking and connectedness to parents and to teachers were significant predictors of experimental smoking when considered independently, but not after accounting for friend and sibling factors. Connectedness to parents and teachers decreased the odds of experimental smoking, while connectedness to friends increased the odds.

Editors' Strategic Implications: This strategy of assessing connectedness to-and smoking behaviors of - friends, siblings, teachers, and parents shows promise in predicting teens' cigarette smoking choices. The finding that the negative effects of unconventional connectedness and smoking by friends and siblings outweigh positive effects of connectedness to adults awaits replication with different samples and measures.

KEY WORDS: connectedness; smoking; rural youth; belonging; risk-taking behavior; mobility.

\footnotetext{
${ }^{1}$ Department of Counseling Psychology, University of Wisconsin - Madison.

${ }^{2}$ Address correspondence to Michael Karcher, College of Education and Human Development, University of Texas at San Antonio, 501 West Durango Blvd., Office 4.314, San Antonio, Texas 78207; e-mail: michael.karcher@utsa.edu.
} 
The literature on factors contributing to adolescent smoking reveals a number of immutable contributors such as the impact that role models for smoking have on youth smoking, as well as the influences of genetic, sex, age, and demographic variables (Brook, Brook, Whiteman, Gordon, \& Cohen, 1990; Bussell et al., 1999; Castro, Maddahian, Newcomb, \& Bentler, 1987; Conrad, Flay, \& Hill, 1992; DeFronzo \& Pawlak, 1994; Krohn, Skinner, Zielinski, \& Naughton, 1989). Several theoretical frameworks have been forwarded to explain adolescents' choice of whether or not to experiment with tobacco, with the two most supported approaches being genetic and social learning theories (Langlois, Petosa, \& Hallam, 1999).

\section{Genetic and Social Learning Theory Explanations of Youth Smoking}

Genetic theories suggest that those youth whose parents smoke are more susceptible to becoming smokers themselves (Bentler, Newcomb, \& Zimmerman, 2002). Yet, genetic explanations suggest that the influence of parents' and siblings' tobacco use reflect the same underlying genetic predisposition and therefore do not see parents and siblings as making separate, unique contributions to youth smoking. Similarly, genetic theories cannot explain sex differences in smoking rates (Conrad, Flay, \& Hill, 1992; DeFronzo \& Pawlak, 1994; Jacobson \& Rowe, 1999).

Social learning theory explanations of experimental smoking link youth's experimental smoking to the social models they have around them who smoke, both in the home and in their peer group (Kandel \& Wu, 1995). From this perspective, youth who begin smoking should report encountering role models who smoke in more contexts than youth who refrain from ever smoking. In the social learning approach, sibling and parental smoking provide two distinct sources of social modeling for smoking, such that both should contribute uniquely to the prediction of which youth will start smoking (Chassin, Presson, Todd, Rose, \& Sherman, 1998). From the social learning perspective, experimental smoking is primarily a function of peer role modeling and vicarious reinforcement that leads youth to expect positive physical and social consequences from smoking. From this perspective, it should be youth who spend the most time with peers and friends who smoke who are the most likely to perceive the positive social benefits of smoking and to experiment with smoking themselves.

\section{The Role of Conventional and Unconventional Connectedness in Experimental Smoking}

A promising concept for understanding youths' relationships in terms of degree of involvement with and caring for others is connectedness (Grotevant \& Cooper, 1998; Youniss \& Ketterlinus, 1987). Connectedness conveys both 
youths' attitudes toward and degree of activity in their larger social ecology and the relationships within it (Karcher, 2001). For example, connectedness to friends captures the amount of time youths spend with their friends as well as how much they care about them. Connectedness therefore reflects how much exposure a youth has to others, which provides a sense of how much opportunity exists for social learning (e.g., for observing friends enjoying smoking). Yet, in previous studies of genetic and role model influences on smoking initiation, rarely have researchers examined how connectedness to others contributes to the likelihood that a youth will begin smoking. Doing so could provide a better understanding of how relationship factors facilitate or impede genetic and social learning processes, and could assist in the development of smoking prevention programs for youth (Bruvold, 1993; Rundell \& Bruvold, 1988).

Connectedness can be characterized as conventional or unconventional depending on whether the activities or people with whom a youth is connected endorse socially sanctioned versus illicit behaviors, like smoking (Jessor \& Jessor, 1977). Conventional relationships, activities, and contexts place social controls against delinquent and illicit behaviors (De Civita \& Pagani, 1996; Pederson, Koval, \& O'Connor, 1997; Turbin, Jessor, \& Costa, 2000). Connectedness to parents and teachers serve as controls against risk taking by encouraging conventional behaviors, beliefs, and attitudes (Bonny, Britto, Klostermann, Hornung, \& Slap, 2000; Hendry \& Reid, 2000; Karcher, 2002). Conversely, connectedness to peers, siblings, and friends more often reflect relationships and behaviors that are not structured or supervised by adults, and therefore place fewer controls or constraints against risk-taking behavior like smoking (Karcher, 2001; Stanton \& Silva, 1992). Therefore conventional and unconventional connectedness should make distinct, indeed opposing contributions to youth smoking (Jessor, 1992).

One way to separate the social learning and genetic influences from the effects of conventional connectedness is to examine the role of connectedness to teachers instead of connectedness to parents. Because students typically have no knowledge of whether or not their teachers smoke and are not genetically related to their teachers, any effects of teacher connectedness on adolescents' experimental smoking would suggest conventional connectedness makes a unique contribution to experimental smoking.

The present study sought to explore the contributions of parental, siblings', and friends' smoking, as well as the additive effects of conventional and unconventional connectedness, on the initiation of smoking among rural adolescents. Given these three theoretical explanations introduced above, several hypotheses were made. First, as a function of either genetic predisposition or role modeling, having parents and siblings who smoke will increase the odds that a youth has experimented with smoking. Second, as a function of vicarious reinforcement, youth whose friends smoke will be more likely to have begun smoking. Third, in addition to the influences of genetics and role modeling as explanatory variables, 
youths' sense of connectedness to both the conventional and unconventional people in their lives also will explain whether or not a youth has experimented with tobacco. Specifically, high levels of unconventional connectedness (to siblings and friends) should increase youths' risk for experimental smoking, but high levels of conventional connectedness (to parents and teachers) should decrease the risk.

\section{METHODS}

\section{Participants}

The sample for this study included 303 middle and high school students from a rural town in the Midwest. Ninety-two percent of the students were Caucasian and $50 \%$ were female. Data were collected from students in two classes in each grade between 6th and 10th. There was an $88 \%$ participation rate. Active consent was used in grades six through eight, and passive consent in grades 9 and 10 per the request of the school administrators. All students completed the Risk and Prevention Questionnaire (Rap-Q) and The Hemmingway: Measure of Adolescent Connectedness during one class period.

\section{Measures}

Risk \& Prevention Questionnaire-Revised (RAP-Q) (Nakkula \& Karcher, 1999; Way, Stauber, Nakkula, \& London, 1994). This survey provided demographic information such as sex, age, socioeconomic status (SES), and how often youth had moved in the past year. Information relevant to youth, peer, parent, and sibling smoking was also collected using this questionnaire. Youth were asked whether they had ever smoked cigarettes (no $=0$; yes $=1$ ). We refer to this binary variable as "experimental smoking" and used it as our main outcome measure. Data on the frequency of smoking for siblings and adults in the house were recorded with responses of never, rarely, sometimes, often, or very often and then were dichotomized into ever vs. never for the logistic regression analyses. Finally, students reported what percentage of their friends smoked cigarettes $(0 \%, 25 \%$, $50 \%, 75 \%$, or $100 \%$ ).

The Hemingway: Measure of Adolescent Connectedness (MAC 4th version; Karcher, 2001). This instrument consists of 72 items designed to measure the adolescents' degree of caring for and involvement in specific relationships. The MAC includes subscales of connectedness to parents, siblings, teachers, and friends. Responses to each of the subscale items use a five-point, Likert-type scale ranging from (1) not true at all, (2) not really true, (3) sort of true, (4) true, to (5) very true. There is one reverse scored item in each subscale. The six items within each of the subscales are averaged to obtain subscale mean scores. 
The Connectedness to Parents subscale measures how much adolescents spend time with their parents and enjoy being with them. The Connectedness to Siblings subscale reflects adolescents' feelings of closeness to and involvement with their siblings. The Connectedness to Friends subscale measures the extent to which the adolescent feels close to and spends time with friends in activities such as talking about personal concerns. The Connectedness to Teachers subscale measures adolescents' efforts to get along with their teachers and their concerns about earning their teachers' respect and trust.

The items in each of the subscales reflect the two primary ways of connecting - through activity or involvement and through caring (e.g., "I spend a lot of time with my friends" and "I enjoy spending time with my brother(s) and sister(s))." Subscales either reflect conventional connectedness to adults (e.g., parents and family subscales) or unconventional connectedness (e.g., friends and sibling subscales).

\section{Statistical Analysis}

To examine the main effects of connectedness, we used a proportional split of the sample for stratification: low $(\leq 3.25)$, medium (3.25-4.25), and high $(\geq 4.25)$. We tested, using Chi-squared statistics, differences in the percentage of youth who reported smoking as a function of their connectedness to parents, teachers, friends, and siblings. Then, multiple logistic regression modeling was used to assess the independent role of smoking by others (friends, parents, and siblings) and of adolescent connectedness (to parents, siblings, friends, and teachers) on experimental smoking. Age, SES, and sex were included in all models to control for potential confounding effects. Additionally, interactions among all variables were tested. Special attention was given to age and sex interactions given previous research findings of sex and developmental differences in smoking initiation and the risk factors involved (Kandel \& Wu, 1995). Statistical significance of logistic regression coefficients was tested using Wald chi-square tests and two-tailed p-values less than 0.05 were considered to indicate statistical significance.

\section{RESULTS}

The prevalence of experimental smoking in this sample of rural youth was $45 \%$. Table I presents characteristics of the sample as well as percentages of experimental smokers across adult, sibling, and friend cigarette use categories. Girls were no more likely than boys to have smoked in this sample. Dramatic increases in the percentage of experimental smokers were found across levels of smoking by parents ("adults in the house"), sibling, and friends which supports both the genetic and social learning theories of experimental smoking. 
Table I. Chi-squared Statistics for Proportional Differences in the Number of Experimental Smokers $(n=135)$ Across Categories and Overall Percent of Participants Who Had Experimented with Smoking $(n=300)$

\begin{tabular}{|c|c|c|c|}
\hline Characteristic & $\chi^{2}(d f)$ & $n$ & $\%$ \\
\hline Sex & $0.4(1)$ & & \\
\hline Male & & 70 & 46 \\
\hline Female & & 65 & 43 \\
\hline Grade & $21.2(4)^{*}$ & & \\
\hline 6 & & 6 & 16 \\
\hline 7 & & 15 & 43 \\
\hline 8 & & 12 & 33 \\
\hline 9 & & 41 & 48 \\
\hline 10 & & 61 & 56 \\
\hline Frequency of sibling smoking & $34.6(4)^{*}$ & & \\
\hline Never & & 69 & 34 \\
\hline Rarely & & 18 & 64 \\
\hline Sometimes & & 10 & 56 \\
\hline Often & & 21 & 84 \\
\hline Very often & & 17 & 63 \\
\hline Frequency of adults in the house smoking & $13.7(4)^{*}$ & & \\
\hline Never & & 61 & 38 \\
\hline Rarely & & 10 & 34 \\
\hline Sometimes & & 8 & 42 \\
\hline Often & & 15 & 48 \\
\hline Very often & & 41 & 64 \\
\hline Percentage of friends that smoke & $57.9(4)^{*}$ & & \\
\hline 0 & & 25 & 23 \\
\hline 25 & & 39 & 43 \\
\hline 50 & & 26 & 49 \\
\hline 75 & & 32 & 84 \\
\hline 100 & & 13 & 93 \\
\hline Parent connectedness & $11.7(2)^{*}$ & & \\
\hline Low & & 43 & 55 \\
\hline Medium & & 54 & 50 \\
\hline High & & 38 & 32 \\
\hline Teacher connectedness & $13.2(2)^{*}$ & & \\
\hline Low & & 60 & 59 \\
\hline Medium & & 38 & 40 \\
\hline High & & 37 & 35 \\
\hline Sibling connectedness & $4.5(2)$ & & \\
\hline Low & & 61 & 52 \\
\hline Medium & & 39 & 40 \\
\hline High & & 35 & 39 \\
\hline Friend connectedness & $4.7(2)$ & & \\
\hline Low & & 19 & 34 \\
\hline Medium & & 51 & 43 \\
\hline High & & 65 & 51 \\
\hline
\end{tabular}

${ }^{*} p<.05$.

There was a significant increase in experimental smoking between 6th and 10 th grades. Given the sharp peak in smoking reported by 7 th graders in this sample (see Table 1), we investigated the ways in which the seventh graders differed from the sixth and eighth graders. The only significant difference was that 
many more 7 th graders had moved one or more times in the past 12 months $(50 \%$ of the 7 th graders had moved vs. $25 \%$ of the students in other grades: $21 \%$ in 6th; $17 \%$ in 8 th; $29 \%$ in 9 th; $25 \%$ in 10 th). Therefore, we included a variable for mobility in later analyses to account for the possibility that mobility was related to smoking or was a surrogate for an important covariate that we did not capture.

There were main effects of several types of connectedness on experimental smoking. Table I presents percentages of experimental smoking across the adolescent connectedness scales stratified into low ( $\leq 3.25)$, medium (3.25-4.25), and high $(\geq 4.25)$. Considering parent and teacher connectedness as measures of conventional connectedness, and friend and sibling connectedness as indicators of unconventional connectedness, the results in Table I indicate that there was a significant decrease in the percentage of experimental smokers at higher levels of conventional connectedness and a trend towards a greater number of experimental smokers among those youth who reported high unconventional connectedness to friends but not to siblings.

The results of subsequent logistic regression modeling to test for the combined effects of the variables in Table I on the likelihood that youth would experiment with smoking are presented in Table II. Model 1 includes only the impact of parental (conventional) factors: parental smoking and connectedness. Both variables were statistically related to experimental smoking. Youth whose parents smoked were 2.34 times more likely to experiment with smoking than those youth whose parents did not smoke. After accounting whether or not a youth's parent smoked or not, youth who were highly connected to their parents were 2.55 times less likely to experiment with smoking than youth who reported low connectedness to their parents.

In model 2, age and teacher connectedness were included as substitutes for connectedness to parents. Because youth are known to report declines in connectedness to parents as a function of adolescent differentiation and identity development processes, the negative relationship between parental connectedness and smoking was confounded by age (Lynch \& Cicchetti, 1997). Therefore, to account for conventional connectedness and age separately, we chose connectedness to teachers to represent conventional connectedness and age to account for the developmental stage of the youth. In addition, the other demographic factors of sex, SES, and whether the student had moved in the past two years were included as predictors.

In model 2, parental smoking, connectedness to teachers, and age were significantly associated with experimental smoking. Youth reporting low connectedness to teachers were at 2.2 times greater risk for experimenting with smoking than youth who reported a moderate connectedness to teachers. The effect of age was an increase in the odds of initiating smoking by $50 \%$ per year. Neither sex nor SES were significant predictors. Having moved in the past year also increased youths' risk for experimental smoking by 2.34 times. 


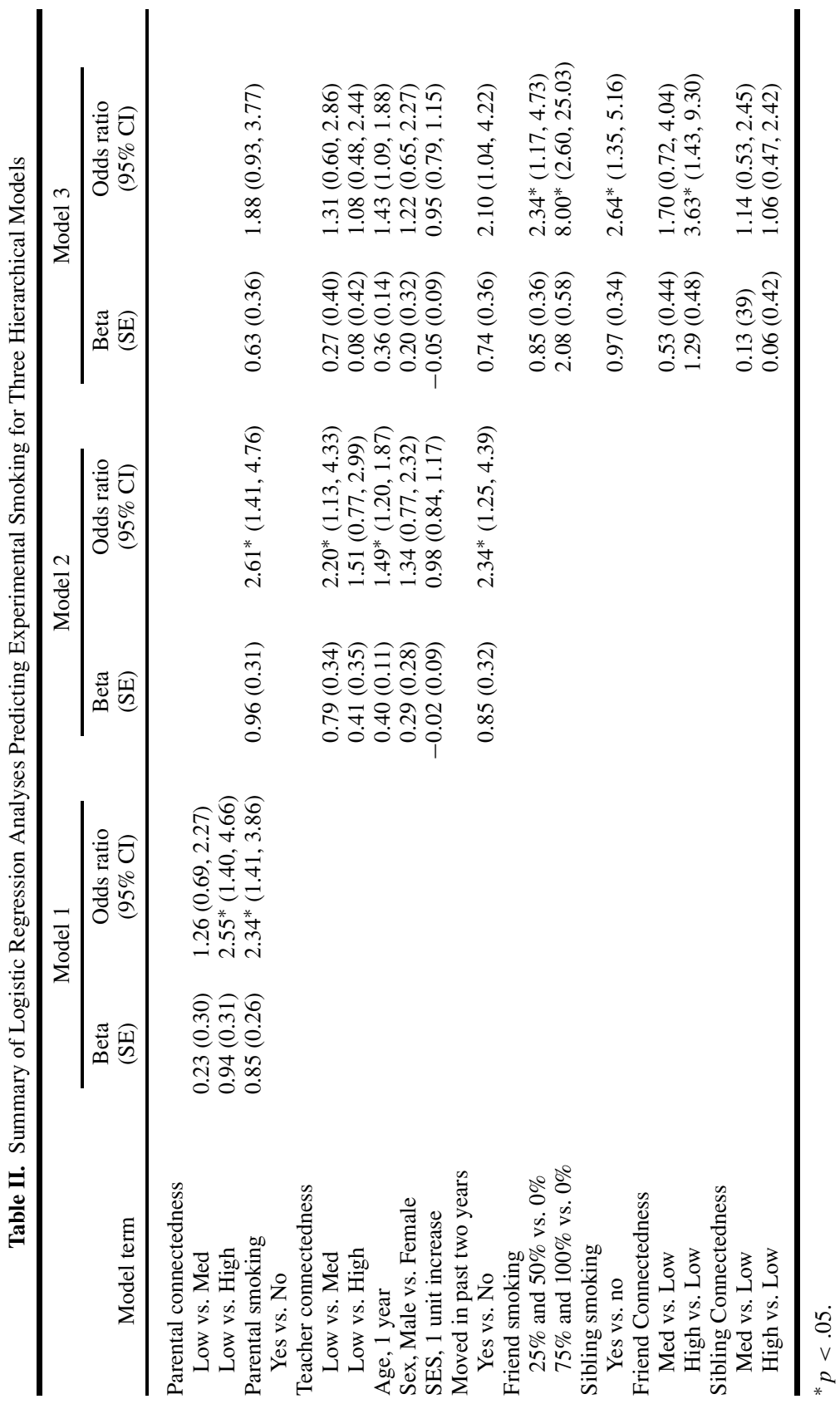


Finally in model 3, the effects of unconventional factors were added in order to test the relative effects of conventional and unconventional variables simultaneously. Friend and sibling smoking as well as connectedness assessments were entered in model 3 as predictors of experimental smoking. Having a high percentage of friends who smoked $(>75 \%$ ) (as compared to no friends who smoked, $<0 \%$ ) increased the odds of youths' experimenting with smoking by eightfold. Even youth who reported that between $25 \%$ and $50 \%$ of their friends smoked were 2.23 times more likely to experiment with smoking than youth who had no friends who smoked. Similarly, having a sibling who smoked increased youths' odds of smoking 2.64 times.

The effects of unconventional connectedness were the opposite of the effects of conventional connectedness. Youth who reported high as compared to low connectedness to friends were 3.63 times more likely to smoke cigarettes. Even youth who reported moderate as compared to low connectedness to friends were 1.7 times more likely to smoke. The effects of sibling connectedness were not significant.

Finally, when these unconventional assessments were entered into the model, the conventional effects of connectedness to teachers and parental smoking became non-significant. A similar model with connectedness to parents also revealed that conventional connectedness had a non-significant effect on the likelihood of youth starting to smoke once the influences of friends and siblings were considered.

\section{DISCUSSION}

The results of this study reveal that connectedness to parents and teachers are protective factors that negatively affect youths' likelihood of starting to smoke, beyond the role of genetic or social learning influences. In addition to the two and a half times increased risk of experimental smoking if one's parents smoked, youth with greater connectedness to parents and to teachers were less likely to start smoking. However, these buffering effects of conventional connectedness became non-significant and trivial in size when the effects of unconventional connectedness were taken into account and when friend and sibling cigarette use were factored into the equation. This suggests that the negative effects of unconventional connectedness and of smoking by friends and siblings overshadows the positive effects of conventional connectedness.

In the final model (3) we found that youth whose parents smoked were 1.88 times more likely to smoke, whose siblings smoked were 2.64 times more likely to smoke, and whose friends smoked were between 2.64 and 8 times more likely to smoke than those whose parents, siblings, and friends did not smoke, respectively. These effects were over and above age effects, which increased youths' risk for smoking by $50 \%$ with each advancing year. In general, higher connectedness to friends was associated with a greatly increased risk of experimental smoking 
compared to those youth who were less connected to their friends regardless of how many of their friends smoked.

\section{The Impact of Conventional and Unconventional Connectedness on Smoking}

This study found support for the hypothesis that the more conventional connectedness youth report the less likely they are to start smoking, and the more unconventional connectedness to friends youth report, the more likely they are to start smoking. This likely is because unconventional connectedness to friends reflects how much time one spends with friends in contexts that tend not to be supervised by adults, allowing for more opportunities to smoke; yet it also may be that the more connected one feels to people in those contexts the more susceptible they are to peer pressure. Conversely, conventional connectedness to parents and teachers, which reflects time spent with and caring for people and places that represent the conventions of society, provides a protective role in the lives of youth. Connectedness to parents and to teachers tends to lessen the likelihood that one will start to smoke, even if the youth's parents smoke.

One surprising finding concerned mobility, which may be viewed as a kind of physical disconnection. Having to move from school to school is one form of disconnection. In this study, having moved doubled the likelihood of experimental smoking (confidence interval between 1.04 and 4.22). It might be argued that such moves compromise connectedness to friends or to teachers, or that it reflects a function of SES or of family dysfunction, yet the effect of having moved was significant even after controlling for SES, sex, age and whether their friends smoked.

\section{Limitations, Future Directions, and Conclusions}

There were several limitations to this study. First of all, we asked about "adults in your home" rather than about specific parents. This was done to include youth who did not live with their parents, but it made the nature of this adult relationship less precise and clear. Another limitation was the self-reported nature of the study. There was no external or objective assessment of youth smoking, smoking in the family, or even of the connectedness that others felt for the youth. There also was the unusual spike in experimental smoking in seventh grade, which disrupted a linear age trend in smoking experimentation. We believe it was a function of the greater number of youth who had moved once or more in the previous year, but we cannot be absolutely sure this was the only or main variable on which the 7 th graders differed. But perhaps the greatest limitations were (a) our inability to assess genetic predisposition more formally (e.g., using a twin design or genetic testing), and (b) that we did not include an assessment of vicarious reinforcement 
related to youths' perceptions of and beliefs about smoking. We could only assume that youth whose parents and siblings smoked were more genetically disposed to start and continue smoking, and that youth whose friends smoked role modeled smoking for the youth in the study (e.g., and not the other way around). Future studies might be able to provide more precise measures of these factors.

The study, however, was able to confirm that in addition to having family or friends who smoke, the nature of one's relationship with those individuals affects youths' likelihood of starting to smoke. Having friends who smoked increased youths' odds of starting to smoke, but in addition to this main effect, regardless of how many of the youths' friends smoked, the more connected youth reported being to their friends, the greater their risk for starting smoking. These unconventional factors, both friends' smoking and connectedness to friends, overshadowed the conventional factors.

In the final analysis, the most important predictors of experimental smoking were having many friends who smoked, being connected to friends, and having parents who smoke, respectively. These results suggest that prevention programs would do well to help youth make better decisions about their affiliation patterns and to help youth understand the possibly adverse consequences of overly intense connectedness to friends. Helping youth better distribute their time among conventional and unconventional contexts and relationships, and helping youth become better at understanding the impact of these social influences in their lives may prove to be powerful prevention strategies.

\section{REFERENCES}

Bentler, P. M., Newcomb, M. D., \& Zimmerman, M. A. (2002). Cigarette use and drug use progression: Growth trajectory and lagged effect hypothesis. In D. B. Kandel (Ed.), Stages and pathways of drug involvement: Examining the gateway hypothesis (pp. 223-253). Cambridge: Cambridge University Press.

Bonny, A. E., Britto, M. T., Klostermann, B. K., Hornung, R. W., \& Slap, G. B. (2000). School disconnectedness: Identifying adolescents at risk. Pediatrics, 106(5), 1017-1021.

Brook, J. S., Brook, D. W., Whiteman, M., Gordon, A. S., \& Cohen, P. (1990). The psychosocial etiology of adolescent drug use and abuse. Genetic, Social, and General Psychology Monographs, 116(2), 111-267.

Bruvold, W. H. (1993). A meta-analysis of adolescent smoking prevention programs. American Journal of Public Health, 83, 872-880.

Bussell, D. A., Neiderhiser, J. M., Pike, A., Plomin, R., Simmens, S., Howe, G. W., et al. (1999). Adolescents' relationships to siblings and mothers: A multivariate genetic analysis. Developmental Psychology, 35, 1248-1259.

Castro, F., Maddahian, E., Newcomb, M., \& Bentler, P. (1987). A multivariate model of the determinants of cigarette smoking among adolescents. Journal of Health and Social Behavior, 28, 273 289.

Chassin, L., Presson, C. C., Todd, M., Rose, J. S., \& Sherman, S. J. (1998). Maternal socialization of adolescent smoking: The intergenerational transmission of parenting and smoking. Developmental Psychology, 34, 1189-1201.

Conrad, K. M., Flay, B. R., \& Hill, D. (1992). Why children start smoking cigarettes: Predictors of onset. British Journal of Addiction, 87, 1711-1724. 
De Civita, M., \& Pagani, L. (1996). Familial constraints on the initiation of cigarette smoking among adolescents: An elaboration of social bonding theory and differential association theory. Canadian Journal of School Psychology, 12, 177-190.

DeFronzo, J., \& Pawlak, R. (1994). Gender differences in the determinants of smoking. The Journal of Drug Issues, 24, 507-516.

Grotevant, H. D., \& Cooper, C. R. (1998). Individuality and connectedness in adolescent development: Review and prospects for research on identity, relationships, and context. In E. Skoe, E. Aspaas, \& A. L. Von der Lippe (Eds.), Personality development in adolescence: A cross national and life span perspective (pp. 3-37). New York: Routledge.

Hendry, L. B., \& Reid, M. (2000). Social relationships and health: The meaning of social "connectedness" and how it relates to health concerns for rural Scottish adolescents. Journal of Adolescence, 23(6), 705-719.

Jacobson, K. C., \& Rowe, D. C. (1999). Genetic and environmental influences on the relationships between family connectedness, school connectedness, and adolescent depressed mood: Sex differences. Developmental Psychology, 35(4), 926-939.

Jessor, R. (1992). Risk behavior in Adolescence: A psychosocial framework for understanding and action. Developmental Review, 12, 374-390.

Jessor, R., \& Jessor, S. L. (1977). Problem behavior and psychological development: A longitudinal study of youth. New York: Academic Press.

Kandel, D. B., \& Wu, P. (1995). The contribution of mothers and fathers to the intergenerational transmission of cigarette smoking in adolescence. Journal of Research on Adolescence, 5, 225-252.

Karcher, M. J. (2001). Measuring adolescent connectedness: Four validation studies. Poster session presented at the annual meeting of the American Psychological Association, San Francisco.

Karcher, M. J. (2002). The cycle of violence and disconnection among rural middle school students: Teacher disconnection as a consequence of violence. The Journal of School Violence, 1(1), 35-51.

Koepke, D., Flay, B. R., \& Johnson, C. A. (1990). Health behaviors in minority families: The case of cigarette smoking. Family Community Health, 13, 35-43.

Krohn, M. D., Skinner, W. F., Zielinski, M., \& Naughton, M. (1989). Elaborating the relationship between age and adolescent cigarette smoking. Deviant Behavior, 10, 105-129.

Langlois, M. A., Petosa, R., \& Hallam, J. S. (1999). Why do effective smoking prevention programs work? Student changes in social cognitive theory constructs. Journal of School Health, 69(8), 326-331.

Lynch, M., \& Cicchetti, D. (1997). Children's relationships with adults and peers: An examination of elementary and junior high school students. Journal of School Psychology, 35(1), 81-99.

Nakkula, M. J., \& Karcher, M. J. (1999). Risk and Prevention Questionnaire-Revised (RAP-QR). Unpublished manuscript, University of Wisconsin-Madison.

Pederson, L. L., Koval, J. J., \& O'Connor, K. (1997). Are psychosocial factors related to smoking in grade-6 students? Addictive Behaviors, 22, 169-181.

Rundell, T. G., \& Bruvold, W. H. (1988). A meta-analysis of school-based smoking and alcohol use prevention programs. Health Education Quarterly, 15, 317-334.

Stanton, W. R., \& Silva, P. A. (1992). A longitudinal study of the influence of parents and friends on children's initiation of smoking. Journal of Applied Developmental Psychology, 13, 423-434.

Turbin, M. S., Jessor, R., \& Costa, F. M. (2000). Adolescent cigarette smoking: Health-related behavior or normative transgression? Prevention Science, 1, 115-124.

Way, N., Stauber, H. Y., Nakkula, M. J., \& London, P. (1994). Depression and substance use in two divergent high school cultures: A quantitative and qualitative analysis. Journal of Youth \& Adolescence, 23(3), 331-357.

Youniss, J., \& Ketterlinus, R. D. (1987). Communication and connectedness in mother- and father-adolescent relationships. Special Issue: Sex differences in family relations at adolescence. Journal of Youth \& Adolescence, 16(3), 265-280. 